\title{
Análisis factorial de las actitudes de niños de 6 a 16 años con dispositivos auditivos hacia la educación inclusiva
}

\author{
Olga María Alegre de la Rosa $\left(^{*}\right)$ y Luis Miguel Villar Angulo $\left(^{* *}\right)$ \\ ${ }^{*}$ ) Universidad de La Laguna (Santa Cruz de Tenerife) - España, $\left.{ }^{* *}\right)$ Universidad de Sevilla - España
}

\begin{abstract}
RESUMEN
Este estudio plantea el problema: ¿qué validez tiene el Cuestionario Incluye para Niños y Adolescentes (CINA) para medir las actitudes de niños y adolescentes con dificultades auditivas hacia su inclusión educativa en centros educativos públicos de las provincias de Gran Canaria y Tenerife? La muestra se compuso de 297 estudiantes de Gran Canaria y 137 alumnos de Tenerife con una media de edad de 11 años. Un análisis factorial exploratorio determinó la confiabilidad y relevancia del CINA. Se hallaron cuatro factores: “Implicación familiar y opinión del uso de la tecnología", "Inclusión en el Centro", "Comunicación con especialistas médicos" y "Valoraciones de la tecnología de apoyo". Existieron diferencias significativas entre estudiantes con implante coclear y audífono en "Inclusión en el Centro" y "Valoraciones de la tecnología de apoyo". La media final de actitudes de estudiantes hacia la inclusión fue alta. El factor "Inclusión en el Centro" tuvo la media más elevada. La media de actitudes de los estudiantes con implante coclear en "Comunicación con especialistas médicos" fue baja.
\end{abstract}

Palabras Clave: Análisis factorial, educación inclusiva, implantes cocleares, niños y adolescentes.

\section{Factorial analysis of the attitudes of children from 6 to 16 years old with hearing devices towards inclusive education}

\section{ABSTRACT}

This study raised the following problem: how valid is the Questionnaire Includes for Children and Adolescents (QICA) to measure the attitudes of children and adolescents with hearing difficulties to educational inclusion in public schools in the provinces of Gran Canaria and Tenerife? The sample consisted of 297 students from Gran Canaria and 137 students from Tenerife with an average of 11 years old. An exploratory factor analysis determined the reliability and relevance of QICA. Four factors were found: "Family involvement and opinion on the use of technology", "Inclusion in the Center", "Communication with medical specialists" and "Support technology assessments". There were significant differences between students with cochlear implants and hearing aids in "Inclusion in the Center" and "Support technology assessments". The final average of students' attitude towards inclusion was high. The factor "Inclusion in the Center" had the highest average. "Communication with medical specialists" was the lowest average attitude of students with cochlear implants.

Keywords: Children and adolescents, cochlear implants, factor analysis, inclusive education.

\section{Introducción}

La pérdida auditiva causada por el daño a la cóclea es la forma más reiterada de pérdida de audición. Aunque se amplíe el umbral de los sonidos (por ejemplo, con un dispostivo auditivo), la percepción de los sonidos es anormal. Las niños y adolescentes que usan dispositivos para aumentar el umbral auditivo tienen dificultades para comprender el lenguaje hablado de sus pares y maestros. Los beneficios de los dispostivos tecnológicos paliativos de la sordera no siempre compensan el problema del desarrollo de la inteligencia y el lenguaje, aunque rubrican actitudes hacia sí mismos, la familia y la sociedad.

\section{Revisión de la literatura}

2.1. Principios organizadores de educación inclusiva para ninos y adolescentes con dificultades auditivas

Los elementos que estructuran la revisión de la literatura de este estudio se ciñen a hipótesis que densifican el conocimiento sobre los niños y adolescentes con dificultades auditivas. Admitimos, igualmente, principios artículadores del bienestar de esos estudiantes bajo supuestos encaminados a la intervención educativa (Moeller, Carr, Seaver, Stredler-Brow, y Holzinger, 2013). Consecuentemene, enunciamos los pilares conceptuales y 
empíricos que originaron el Cuestionario Incluye para Niños y Adolescentes (CINA) de este estudio con objeto de conocer las percepciones de niños y adolescentes con dificultades auditivas sobre la inclusión educativa en las escuelas públicas de dos provincias de Islas Canarias.

\subsection{Intervención y participación familiar}

Una de las conjeturas básicas en la educación de niños y adolescentes con dificultades auditivas es que las familias, los docentes y los profesionales vinculados provoquen un ambiente óptimo que facilite el dominio del lenguaje para el desarrollo intelectual y rendimiento académico de esos estudiantes.

En los procesos postimplantación de una tecnología acústica, los padres asumen el doble rol de aprendices o maestros, o la dominancia de uno de esos roles en función de la inseguridad percibida o de la responsabilidad atribuida.

Teniendo presente que la escuela es una institución que provee servicios a la sociedad, cualquier proyecto educativo tiene la obligación de "interactuar de manera respetuosa con la cultura de las familias, creencias y actitudes" (Moeller, Carr, Seaver, Stredler-Brown, y Holzinger (2013, pp. 436-437) para que los estudiantes con dificultades auditivas aprendan a confrontarlos con numerosas oportunidades de interacción comunicativa que normalicen su participación activa en situaciones escolares y extraescolares.

\subsection{Trabajo colaborativo en equipo en un centro escolar}

Un proyecto educativo de centro que asegure como principio la inclusión, ha de apoyar a las familias en la detección auditiva y la implantación de esquemas de expresión hablada en niños y adolescentes recién implantados. Cuanto más evidente se hace la necesidad de comunicación de niños y adolescentes con dificultades auditivas, más dificil se nos presenta la realidad de centros educativos poco accesibles y sin "interacciones de compañeros y oportunidades de amistad e interacciones sociales" (Schick, et al.,; Patrick, 2013, p. 48). Por ello, se deben considerar acciones prácticas en el ámbito escolar que disipen las vacilaciones sobre la naturaleza inclusiva en los contextos educativos. Davenport y Alber-Morgan (2016) llamaron la atención sobre ciertos aspectos constructivos de los edificios que incrementaban la rumorosidad en la comunicación y disminuían la atención de los niños con dificultades de audición: "Pisos de baldosas, muros de hormigón, y grandes ventanas que reverberan el sonido" (p. 44).

Mientras que la cultura anglosajona ensanchaba el conocimiento del lenguaje y la comunicación de los estudiantes con dificultades auditivas, el estudio de Scott y Kasun (2018) se centraba en el análisis de la lengua de signos como primera línea de comunicación para que los niños sordos interactuaran con el mundo, instando los maestros al uso de recursos y prácticas (instrucción explícita, andamiaje, creación de conocimientos previos e instrucción individualizada) para satisfacer las necesidades de estudiantes y promover la igualdad de oportunidades y la justicia social.

Los centros educativos que apoyan una educación inclusiva para niños que usan dispositivos auditivos, supuestamente ofrecen un currículo apropiado para sus respectivas edades con adaptaciones curriculares para mejorar el rendimiento académico (Chute y Nevins, 2006). Bajo estos supuestos teóricos funcionan los proyectos educativo y curricular de centro con una filosofía basada en la esencia de la participación en clase y centro, colaboraciones con pares y maestros, decisiones sobre relaciones, importancia de las competencias emocionales de los estudian- tes y desarrollo de su autoconcepto (Cepa, Heras, y Fernández-Hawryla, 2017). Este conjunto de razonamientos nuclearon el proyecto interdisciplinar de acondicionamiento de un espacio escolar en el estudio de Amiama, Ledesma, y Monzón (2017) para facilitar la inclusión de todo el alumnado de $3^{\circ}$ de ESO.

La autoeficacia docente alude a la habilidad personal de un maestro. Un profesor autoeficaz en un ambiente inclusivo, de acuerdo con la literatura sintetizada por Sharma, Jitoko, Macanawai y Forlin (2018, p. 14), tiene un "conocimiento de contenido y pedagogía", adopta un modo de "gestión de ambiente de clase y de conducta" y manifiesta una "habilidad para trabajar colaborativamente con padres y paraprofesionales" e incluso se asocia con el éxito de estudiantes.

A Radici, Heboyan, Mantovani, y De Leo (2018) les bastó aplicar el cuestionario Teacher Attitude Scale para calibrar comprobar las debilidades que afectaban las percepciones de maestros sobre sí mismos y de los alumnos que usaban tecnologías de comunicación aumentativa y alternativa.

\subsection{La implantación de dispositivos electrónicos}

Los resultados de la implantación auditiva varían en niños y adolescentes, y consecuentemente, aumentan la variabilidad entre ellos. Las percepciones de esos estudiantes desvelan el grado de satisfacción con aquellos factores educativos que más influyen la inteligibilidad en la comunicación hablada (Moore, 2008).

Existen diferencias entre estudiantes que usan implantes cocleares, en función de la fecha de implantación. De ahí que se hayan realizado estudios evaluativos sobre la calidad de vida en los estadios prequirúrgico y durante la rehabilitación postquirúrgica, así como de la inclusión de estudiantes en centros escolares que manifiesten habilidad auditiva y de cómo esta destreza facilita o bloquea la comunicación hablada (Noble, 2013).

\subsubsection{Edad de implantación y acceso oportuno a servicios edu- cativos}

El problema de la edad de implantación auditiva en estudiantes se ha asociado con otras variables como el lenguaje expresivo (Tomblin, Barker, Spencer, Zhang, y Gantz, 2005), la interacción materna (Bakar, Brown, y Remine, 2010), o la adecuación de las puntuaciones del lenguaje a la edad (Geers, Nicholas, Tobey, y Davidson, 2016). No sorprende que Holt, Beer, Kronenberger, Pisoni, y Lalonde (2012) estudiaran las relaciones entre el ambiente familiar (medido a través de la Family Environment Scale) y obtuvieran que parte de la variabilidad en el desarrollo de los estudiantes con implantación coclear se relacionaba con el ambiente familiar.

\subsection{Tecnologías de asistencia para niños y adolescentes con pérdidas auditivas}

La primera función de los dispositivos tecnológicos es restaurar la audición por vía de la selección de una frecuencia y de su amplificación. La información visual acompañante a la identificación del habla ha sido un problema recurrente entre autores, al entender que las claves del habla visual son formas bajo las cuales los estudiantes sordos o con dificultades auditivas pueden acceder a una señal hablada.

\subsubsection{Apoyo de modos de comunicación}

Los modos hablados de comunicación o por medio de signos constituyen un asunto reiterado en la investigación de es- 
tudiantes implantados. Hyde y Punch (2011) investigaron ambos modos en Australia sondeando a padres, maestros y niños, y averiguaron que el lenguaje de signos (inglés o australiano) apoyaba el desarrollo personal, social y académico de escolares implantados.

La comunicación de los estudiantes con implantes fue básica para el estudio de_Gale (2011), que analizó la lengua de signos y el habla usando textos narrados suscribiendo que los "usuarios con implantes cocleares pueden ser bilingües usando ambos lenguajes, oral y visual" (p. 136).

El lenguaje de signos parece aconsejable, no obstante, en ambientes ruidosos de clase. Así, el estudio de Caldwell y Nittrouer (2013) obtuvo dos resultados sorprendentes: los efectos del ruido fueron consistentes entre los grupos de niños oyentes y con implante coclear y, sin embargo, los puntajes en otras medidas no explicaron ninguna diferencia grupal en el reconocimiento de la voz.

\subsection{Evaluación de estudiantes con pérdida auditiva}

No resulta extraña la proliferación de escalas evaluativas destinadas al profesorado, familias y estudiantes con dificultades auditivas para ayudar a los escolares en su desarrollo (Bradham y Houston, 2014). En este sentido, advertía Bellis (2011, p. 229): "Una evaluación integral del procesamiento auditivo central debe incluir pruebas de más de una categoría...".

\subsubsection{Auto-valoración de percepciones y actitudes}

¿Cuáles son las actitudes (integradas por componentes cognitivos, afectivos y conductuales) que tienen los niños y adolescentes sordos y con dificultades de audición respecto de sus compañeros con o sin dificultades físicas o mentales?

El estudio de las actitudes de estudiantes con dificultades de aprendizaje se ha apoyado en distintas teorías psicológicas: a) la teoría de la acción razonada que investiga los determinantes de las actitudes de niños de educación primaria y las intenciones de comportamiento que tienen hacia compañeros con discapacidades físicas, medida a través de Peer Attitudes Toward the Handicapped Scale y de Behavioural Intention Scale (Roberts, y Lindsell, 1997); b) la teoría del contacto (experiencias de contacto, cercanía y normas de clase) y la teoría ecológica del desarrollo humano (configuración de clase, nivel de grado y género) para conocer las perspectivas que tienen estudiantes de la inclusión de compañeros de clase sordos o con dificultades auditivas, medidas por medio de cuatro escalas (Inclusion, Contact, Closeness, y Class Norms) (Hung, y Paul, 2006); c) teoría de campo de la Gestalt y teoría dialógica de las autopercepciones para conocer las autoconfiguraciones de estudiantes con dificultades leves de aprendizaje, que Grobler, y Wessels, 2018 avalaron en un estudio cualitativo, subrayando que si los maestros invierten tiempo con estudiantes, éstos se sienten valorados, confiados y exprimentan una sensación de dignidad.

La medición de actitudes de niños con dificultades físicas e intelectuales ha sido objeto de múltiples estudios, que Nowicki, y Sandieson (2002) metaanalizaron revelando que los niños preferían compañeros sin dificultades físicas e intelectuales. Las actitudes de rechazo, la falta de amistades o los sentimientos de aislamiento las experimentan más acusadamente los niños con dificultades intelectuales que quienes no las tienen (Beaulieu-Bergeron, y Morin, 2016).

En el contexto cultural israelí, Most, Wiesel, y Blitzer (2007) investigaron las orientaciones de adolescentes sordos y con dificultades auditivas hacia el implante coclear y averiguaron que los participantes expresaron actitudes positivas hacia la tecnología del implante coclear.

Para tener éxito con las tecnologías de audición, los usuarios deben poseer autoconfianza, autoestima, extroversión y locus de control, según Rekkedal (2012), que llegó a identificar aquellos factores que promovían el uso de ayudas de audición (pérdida severa de audición, actitudes positivas y calidad de sonido de dispositivos).

El autoconcepto y el desarrollo del yo, son dos aspectos interrelacionados del bienestar autoindicativo y la maduración sociocognitiva. El estudio de Van Gent, Goedhart, Knoors, Westenberg, y Treffers (2012) mostró que los adolescentes sordos autopercibieron niveles bajos de aceptación social, amistades cercanas, desarrollo del yo y mayor apariencia física. Además, mantuvieron una autoestima positiva global durante la infancia, calidad de comunicación con padres y desarrollo del yo asistiendo regularmente a un centro escolar.

Trabajando las percepciones de autoeficacia de adolescentes sordos, Michael, Cinamon, y Most (2015) compararon tres grupos de adolescentes de educación secundaria y bachillerato averiguando que los participantes sordos notificaron niveles significativamente más altos de claridad hacia su futuro que los otros dos grupos con dificultades auditivas y oyentes.

\subsection{Estructura factorial y validación de cuestionarios para una educación inclusiva}

Estudios previos han utilizado la metodología del análisis factorial confirmatorio para analizar la estructura interna de cuestionarios administrados a niños y adolescentes para una educación inclusiva. De este modo procedieron Alegre y Villar (2015) con el cuestionario Actitudes hacia la Inclusión e Interculturalidad (CAII) dilucidando su estructura factorial subyacente.

De otra parte, se ha estudiado la estructura factorial interna del cuestionario KINDL para comprobar los estándares de calidad de vida en niños y adolescentes asturianos con dificultades auditivas (Fernández-López, Fernández-Fidalgo, Cieza y Ravens-Sieber, 2004). En otro contexto, Navarro y Merino (2016) aplicaron el análisis factorial confirmatorio a las respuestas de KINDL de una muestra de niños peruanos y obtuvieron menos factores que la versión original alemana de $K I N D L$, pero con mayor consistencia interna.

El Cuestionario de Fortalezas y Debilidades ( $S D Q$ ) se ha usado ampliamente en niños y adolescentes con problemas de audición. La primera provisión que hicieron los investigadores con $S D Q$ consistió en evaluar su estructura factorial. Así lo hicieron, entre otros, McAloney-Kocaman y McPherson (2017) averiguando la composición de cinco factores de $S D Q$ mediante un análisis factorial confirmatorio de mínimos cuadrados.

\subsection{Problema}

Dada la importancia otorgada a las actitudes de niños y adolescentes a la educación inclusiva, planteamos la siguiente cuestión: ¿qué validez y fiabilidad tiene el Cuestionario Incluye para Niños y Adolescentes (CINA) para medir las actitudes de niños y adolescentes con dificutades auditivas a la inclusión educativa en centros escolares públicos de las provincias de Gran Canaria y Tenerife? El estudio trata de analizar la estructura subyacente a una serie de ítems del CINA para interpretar los factores que explican por qué unos ítems se relacionan más con unos que con otros. 


\section{Método}

\section{Participantes}

La muestra de niños y adolescentes participantes con dificultades auditivas y sin otros déficits asociados fue de 297. Los estudiantes asistieron a centros educativos ordinarios de Islas Canarias. Atendiendo al género, 161 (54.2\%) fueron niños y 136 $(45,8 \%)$ niñas. La media de edad fue de 11,07 años $(\mathrm{sd}=3,61)$. En función del dispositivo, 187 (62,3\%) utilizaban implante coclear (IC) y 110 (37\%) audífonos (AU). Cursaban Educación Infantil $(n=30,10,1 \%)$, Educación Primaria $(n=152,51,2 \%)$, Educación Secundaria Obligatoria ( $n=104,35 \%)$, y Bachillerato o Formación Profesional ( $\mathrm{n}=11,3,7 \%)$. Tenían Adaptaciones Curriculares Individualizadas (ACIs) $(\mathrm{n}=118,39,7 \%)$, frente a quienes no tenían ACIs ( $n=179,60,3 \%)$. El sistema de comunicación con sus familias era oral $(n=212,71,4 \%)$, bilingüe $(n=57,19,2 \%)$ o por lengua de signos $(n=28,9,4 \%)$. La media de edad de diagnóstico de la sordera fue antes de un año $(\mathrm{sd}=0,942)$ siendo la causa prenatal la mayoritaria $(n=171,57,6 \%)$, seguida de causa desconocida $(n=$ $66,22,2 \%)$, perinatal $(n=35,11,8 \%)$ y posnatal $(n=25,8,4 \%)$. Eran estudiantes con valores medios (Med=3,86, $\mathrm{sd}=0,903$ ) medidos por el Test de Raven - Matrices Progresivas (entre 26-75 cociente intelectual); valores medios ( $\mathrm{Med}=2,80, \mathrm{sd}=1,25$ ) evaluados por el Peabody. Test de Vocabulario en Imágenes, y moderadamente bajos, según valores obtenidos en el Test Illinois de Aptitudes Psicolingüísticas (ITPA) (Med=2,45, sd=0,739).

\section{Procedimiento}

Se solicitó al equipo especialista de atención a dificultades auditivas del Gobierno de Canarias la identificación de aquellos niños y adolescentes que cumplían los requisitos del estudio en cuanto a edades, uso de dispositivos auditivos y no tener otros déficits asociados a la sordera. Previa autorización escrita de los padres, se procedió a administrar el Cuestionario Incluye para $\mathrm{Ni}$ ños y Adolescentes (CINA) con el apoyo de los profesores tutores de aula que facilitaron el espacio para que logopedas profesionales entrenados pudieran comunicarse con los niños y adolescentes con dificultades auitivas y que estos cumplimentaran el cuestionario.

\section{Instrumentación}

El Cuestionario Incluye para Niños y Adolescentes (CINA) fue elaborado por los investigadores para este estudio siguiendo el modelo proyectado por Booth y Ainscow (2002) para el Index for Inclusion. Se elaboró un primer cuestionario que se envió a expertos en audición y lenguaje, y en inclusión, que hicieron de jueces matizando terminologías y conceptos. Posteriormente, se administró a un grupo de 10 niños sordos de tres centros educativos privados (no participantes en el estudio) con objeto de conocer su posible dificultad comprensiva mejorándose la calidad y adecuación de los ítems. El cuestionario consta de 40 items agrupados en tres dimensiones. El modo de respuesta fue mediante una escala Likert de 4 puntos que oscilaban desde "nunca" a "siempre" en la primera dimensión vinculada a la frecuencia de las afirmaciones presentadas relacionadas con la implicación familiar (items 1-10). La segunda dimensión se relacionaba con el grado de acuerdo ("totalmente en desacuerdo" a "totalmente de acuerdo") con afirmaciones relacionadas con la inclusión en el centro, los apoyos y la comunicación con los profesionales (items 11-28). Finalmente (items 29-40) se solicitaba la opinión (items 29-34) sobre el uso de la tecnología de apoyo ("nunca" a "siempre") y la valoración sobre dicha tecnología (items 3540) con respuestas que fluctuaban de "muy adecuado" a "muy inadecuado".

Los 40 ítems del CINA tienen concomitancias con ítems de los siguientes instrumentos: Peer Attitudes Toward the Handicapped Scale, que ofrece puntuaciones en tres subescalas: "dificultades físicas, de aprendizaje y de comportamiento", y Behavioural Intention Scale, que "evalúa cómo se comportarían los estudiantes hacia un compañero hipotético con una minusvalía física" (Roberts, y Lindsell, 1997, pp. 137-138); Inclusion, Contact, Closeness, and Class Norms, que contiene ítems en "cuatro subescalas: (Inclusion, Contact, Closeness, y Class Norms) y una información general" (Hung, y Paul, 2006, p. 65); Classroom Participation Questionnaire, usado para obtener información de la participación individual de un alumno en la enseñanza general de clase (Antia, Sabers, y Stinson, 2007); Deaf Identity Development Scale (DIDS), que aísla cuatro subescalas: "identidad de sordos (inmersión), identidad auditiva, identidad bicultural e identidad marginal" (Most, Wiesel, y Blitzer, 2007, p.73); Family Environment Scale, que evalúa tres dimensiones: “(a) relaciones familiares, (b) crecimiento personal y metas dentro de la familia, y (c) enfoque de la familia en el mantenimiento del sistema" (Holt, Beer, Kronenberger, Pisoni, y Lalonde, 2012, p. 852), y Teacher Attitude Scale, que mide las actitudes de los profesores hacia los niños que utilizan la comunicación aumentativa y alternativa (AAC) (Radici, Heboyan, Mantovani, y De Leo, 2018), entre otos instrumentos.

\section{Análisis de datos}

Se analizaron los datos a través del análisis factorial exploratorio (AFE) utilizando el "Statistical Package for Social Sciences (SPSS)" para determinar grupos de variables significativas latentes existentes entre ítems del CINA. AFE permitió la reducción del tamaño de un banco de ítems con objeto de identificar las dimensiones básicas (Deng, Wang, Guan, y Wang, 2017).

\section{Resultados}

Mediante la metodología cuantitativa se llevó a cabo un análisis de la adecuación de la muestra de Kaiser-Meyer-Olkin, cuyo valor obtenido (0.806) permitió considerar el uso del AFE. El test de esfericidad de Bartlett confirmó el resultado anterior, que fue significativo $\left[\mathrm{X}^{2}(780)=5734,769, p<0,001\right]$, indicando que la muestra se adecuaba globalmente e ítem a ítem a la realización del AFE. El alpha de Cronbach dio un coeficiente de 0.882, que maximizaba la generalizabilidad de los factores. El alfa de Cronbach de manera diferenciada fue de 0.863 para los niños con IC y de 0,927 para los que usaban AU. Se examinó el porcentaje de varianza total que explicaba cada factor, obteniéndose que cuatro factores explicaban el 55,816 de dicha varianza.

Las comunalidades fueron en general elevadas llegando a 0.843, implicando que la mayoría de los ítems estaban bien representados en el espacio de los factores.

Una vez realizado el AFE, se simplificó la información mediante la matriz de correlaciones, con objeto de conocer la estructura subyacente a los items del CINA. La matriz rotada, según el método ortogonal varimax de máxima verisimilitud, permitió agrupar los ítems con mayor peso en cada factor. Teniendo en cuenta el tamaño de la muestra y la facilidad de interpretación de las variables, se eligió una carga $>0,40$ para los factores, según el corte planteado por Yong y Pearce (2013).

Para llevar a cabo la rotación de los factores se aplicó el principio de estructura simple planteado por Thurstone (1928) obteniéndose cuatro factores (Tabla 1 ): 
Factor 1. "Implicación familiar y uso de tecnología" tiene un poder explicativo del 14,038\% de la varianza total, obteniendo el mayor peso el ítem ("Tu padre es el que te ayuda en las tareas de casa") que representa el 67,24\% (0.820), seguido de ("Tu padre es el que viene al colegio/instituto a las reuniones") que representa el $61,15 \%$ (0.782).

Factor 2. "Inclusión en el centro" supone el 13,527\% de la varianza total. El ítem "La atención médica que recibo en el centro es adecuada" supone el 63,36\% (0.796), seguido del ítem "Cuento con profesorado suficientemente preparado", cuyo peso representa el $45,02 \%(0.671)$.
Factor 3. “Comunicación con especialistas médicos en el caso de niños implantados" representa el 11,43\% de la varianza, siendo el ítem de mayor peso "El equipo médico te informó después del implante de manera detallada del resultado de la implantación", que representa el 89,49\% (0.946), seguido del ítem “El equipo médico te informó antes del implante de manera detallada del resultado de la implantación" con 89,30\% (0.945).

Factor 4. "Valoraciones sobre la tecnología de apoyo recibida". El factor engloba el 8,057\% de la varianza total. El ítem de mayor peso fue "Valoración tecnología en el aula: apoyos visuales" que representa el 81\% (0.900), seguido del ítem "Valoración tecnología en el aula: pizarra digital" que supone el 76,38\% (0.874).

Tabla 1

Factores obtenidos en el CINA

\begin{tabular}{|c|c|c|c|c|}
\hline \multirow[t]{2}{*}{ Items } & \multicolumn{4}{|c|}{ Factores } \\
\hline & 1 & 2 & 3 & 4 \\
\hline 10. Implicación familiar: tu padre es el que te ayuda con las tareas de casa & ,820 & & & \\
\hline 9. Implicación familiar: tu padre y es el que viene al colegio/instituto a las reuniones & 782 & & & \\
\hline 1. Implicación familiar:tu padre y/o madre revisan tus tareas escolares & ,683 & & & \\
\hline 29.Opinión uso tecnología: bucle magnético & ,642 & & & \\
\hline 5. Implicación familiar: tu padre y/o madre asisten y participan en las actividades de tu centro escolar & 639 & & & \\
\hline 30.Opinión uso tecnología: sistema roger & ,632 & & & \\
\hline 4. Implicación familiar: tu padre y/o madre solicitan reuniones con tus tutores &, 559 & & & \\
\hline 32.Opinión uso tecnología en el aula: pizarra digital &, 549 & & & \\
\hline 33.Opinión uso tecnología en el aula: presentaciones con imágenes &, 541 & & & \\
\hline 8. Implicación familiar: tu madre es la que te ayuda con las tareas en casa &, 539 & & & \\
\hline 19.Inclusión en el centro: la atención médica que recibo en el centro es adecuada & & ,796 & & \\
\hline 17.Inclusión en el centro: cuento con profesorado suficientemente preparado & & 671 & & \\
\hline 21.Inclusión en el centro: los apoyos de especialistas que tengo en el centro favorecen mi mejora comunicativa & & ,656 & & \\
\hline 11.Inclusión en el centro: el centro al que acudo hace que me sienta acogido & & ,637 & & \\
\hline 14.Inclusión en el centro: el centro se esfuerza en disminuir las prácticas discriminatorias & & ,601 & & \\
\hline 20.Inclusión en el centro: los apoyos tecnológicos que tengo me ayudan en mi comunicación & & ,599 & & \\
\hline 12.Inclusión en el centro: el centro tiene expectativas altas hacia mi & & ,566 & & \\
\hline 13.Inclusión en el centro: el profesorado piensa que todo el alumnado es igual de importante & &, 500 & & \\
\hline $\begin{array}{l}\text { 24.Comunicación: (si estás implantado) el equipo médico te informó después del implante de manera detallada del } \\
\text { resultado de la implantación }\end{array}$ & & & ,946 & \\
\hline $\begin{array}{l}\text { 23.Comunicación: (si estás implantado) el equipo médico te informó antes del implante de manera detallada del resul- } \\
\text { tado de la implantación }\end{array}$ & & & ,945 & \\
\hline $\begin{array}{l}\text { 25.Comunicación: (si estás implantado) la comunicación que tengo con el equipo médico es satisfactoria porque me } \\
\text { hablan de manera clara, directa y comprensible }\end{array}$ & & & ,643 & \\
\hline 40.Valoración tecnología en el aula: apoyos visuales & & & & ,900 \\
\hline 38.Valoración tecnología en el aula: pizarra digital & & & & ,874 \\
\hline 39.Valoración tecnología en el aula: presentaciones con imágenes & & & & ,863 \\
\hline
\end{tabular}

Con los factores obtenidos se buscó obtener una media general que reflejara la actitud de los estudiantes hacia la inclusión, incluyendo, para el caso de los niños y adolescentes con IC, el factor 3 relativo a la comunicación con especialistas médicos.

La media final de la actitud de los estudiantes hacia la inclusión (factor 1, 2 y 4) fue de 2,74 que, en un rango de 1 a 4 (de muy baja a muy alta) supone una actitud cercana a 3 , es decir, una valoración alta hacia la inclusión. Si se incluye el factor 3 que sólo afectó a los estudiantes con IC, se obtuvo una actitud general baja (Med=1,80).

El factor 3 alcanzó la media más baja ( $\mathrm{Med}=1,96, \mathrm{sd}=0,830)$, seguido del factor $4(\mathrm{Med}=2,51, \mathrm{sd}=1,285)$. El factor 2 tuvo la media más elevada (cercano a la valoración alta) $(\mathrm{Med}=2,92, \mathrm{sd}=$ $0,745)$ seguido del factor 1 (Med=2,77, $\mathrm{sd}=0,725$ ).

Realizado un análisis de la varianza para cada uno de los cuatro factores obtenidos, diferenciando aquellos que emplea- 
ban IC o AU, se hallaron diferencias significativas entre ambos grupos para el factor 2 y 4, con medias más altas en el caso de los estudiantes con IC que quienes usaban AU (factor 2 med $_{\mathrm{IC}}=3,31$; $\left.\operatorname{med}_{\mathrm{AU}}=2,29\right)\left(\right.$ factor $\left.4 \operatorname{med}_{\mathrm{IC}}=3,30, \operatorname{med}_{\mathrm{AU}}=1,23\right)$. En el factor 3, los estudiantes que usaban IC tuvieron diferencias significativas intragrupo $(t=32,13, p<0,001)$, vinculadas a edad, sexo y otras variables relativas a edad del implante. Finalmente, no se obtuvieron diferencias significativas entre los que usaban IC o AU, en implicación familiar, y uso y valoración de la tecnología de apoyo a dificultades auditivas (Tabla 2).

Tabla 2

Valores de la t de Student para cada uno de los cuatro factores obtenidos en el CINA

\begin{tabular}{|c|c|c|c|c|c|}
\hline \multirow[t]{2}{*}{ Factores } & \multirow{2}{*}{$\begin{array}{c}t \\
t \text {-Student, } \mathrm{p}<\end{array}$} & \multicolumn{2}{|c|}{ IC $(n=184)$} & \multicolumn{2}{|c|}{$\mathrm{AU}(\mathrm{n}=113)$} \\
\hline & & Med & sd & Med & sd \\
\hline 1. Implicación familiar y opinión del uso de la tecnología & -.905 n.s. & 2,77 & ,725 & 2,85 & ,752 \\
\hline 2. Inclusión en el Centro & $15,38^{* * *}$ & 3,31 &, 543 & 2,29 &, 575 \\
\hline 3. Comunicación con especialistas médicos & $32,13^{* * *}$ & 1,96 & ,830 & & \\
\hline 4. Valoraciones de la tecnología de apoyo & $21,437^{* * *}$ & 3,30 & ,965 & 1,23 & ,430 \\
\hline
\end{tabular}

\section{Discusión}

Los hallazgos de este estudio indican que el CINA es una herramienta que mide las actitudes de niños y adolescentes con dificultades auditivas en centros escolares públicos de educación inclusiva de Gran Canaria y Tenerife.

El primer factor "Implicación familiar y opinión del uso de la tecnología", contiene 10 ítems sobre implicación familiar (asistencia y solicitud de reuniones, ayudas a las tareas y valoración del rendimiento) y 5 sobre el uso de la tecnología (bucle magnético, sistema roger, pizarra digital, imáges y representaciones, y apoyos visuales). La media obtenida refleja una actitud general de estudiantes cercana a la valoración favorable o alta.

Resulta sorprendente que los ítems relativos al uso de la tecnología se integren dentro del factor relativo a la familia. Interpretamos que se debe a que las familias suelen tener especial implicación con el buen uso de dichos dispositivos tecnológicos y los hijos los vinculan con la implicación familiar. Sin embargo, consideramos que estos ítems han de analizarse con mayor profundidad en futuros estudios.

La realidad del implante y la sordera en el ámbito social coloca a las familias ante situaciones de inquietud y desaliento (Hintermair, y Albertini, 2005); Mouvet, Matthijs, Loots, Taverniers, y Van Herreweghe (2013) sugieren que un niño se beneficia del enfoque bilingüe hasta los 9 meses de edad. Después de la implantación, aparecen tendencias hacia un enfoque monolingüe. La provisión de información sobre la intervención temprana a estudiantes con dificultades auditivas es complicada por los elementos emocionales implicados en los procesos decisionales entre la gestión de los profesionales y las opciones seleccionadas por los padres (Kecman, 2018). A causa del estrés familiar producido por la sordera, los especialistas proporcionan a las familias "información sobre todas las opciones y tecnologías disponibles, así como evidencia sobre los beneficios (ventajas) y daños (desventajas) de cada opción para respaldar sus elecciones" (Ching, et al., 2018, p. 24).

Designamos "Inclusión en el Centro" al segundo factor (10 ítems). Alude al personal (médicos, tutores y especialistas), centro (acogida, expectativas y apoyos tecnológicos), valores (igualdad y justicia social), y socialización (participación en actividades de clase y extraescolares).
Una evaluación interdisciplinaria de niños con dificultades auditivas permite la identificación y tratamiento de trastornos significativos de oftalmología, neurodesarrollo, genéticos y del habla y lenguaje (Wiley, Arjmand, Meinzen-Derr, y Dixon, 2011). En posteriores experimentos se ha averiguado que los niños con dificultades auditivas tienen una mayor probabilidad de tener dificultades en su desarrollo, por lo que las evaluaciones tempranas del desarrollo son útiles para la posterior intervención (Meinzen-Derr, Wiley, Phillips, Altaye, y Choo, 2017).

De otra parte, Garberoglio, Gobble y Cawthon (2012, p. 378) señalaron que la formación de maestros y el ambiente escolar priorizan "estrategias instructivas y de gestión de clase frente a la implicación de los propios estudiantes" subrayando que la percepción de eficacia colectiva de maestros sustentaba y cohesionaba el sentido de eficacia docente. Una percepción de eficacia que debe iniciarse preparando a los maestros en competencias críticas para ser eficaces: "preparación profesional, defensa y flexibilidad" (Woolf, 2018, p. 10). Una flexibilidad que debe pasar por el aumento de la participación de los niños con dificultades auditivas en las actividades (Amiama, Ledesma, y Monzón, 2017; Antia, Sabers, y Stinson, 2007).

Denominamos "Comunicación con especialistas médicos" al tercer factor que alude a la comunicación del equipo médico con los estudiantes antes y después del implante, así como la satisfacción de los mismos con el equipo médico por la información clara ofrecida. La media baja en este factor expresa que los niños y adolescentes con IC no reflejan una actitud favorable o de satisfacción con la comunicación que han tenido con los profesionales médicos antes y después del implante coclear.

Se ha estudiado el impacto del implante coclear en procesos de integración educativa, comunicación y rendimiento académico de estudiantes, y se ha comprobado que la implantación coclear temprana mejora el lenguaje hablado y el rendimiento educativo (Alegre, Rodríguez, Villar, y Pérez, 2016). No obstante, existen retos en la educación inclusiva vinculados a aspectos de desarrollo profesional docente, manejo de recursos y nuevas tecnologías, y creación y mantenimiento de un ambiente físico adecuado (Archbold, y Mayer, 2012). Los retos son incitaciones para la creación de sistemas sólidos de intervención temprana, cuando se ha confirmado un diagnóstico audiológico y se ha procurado una información adecuada, individualizada, específica 
y de alto nivel. Entonces se ponen en funcionamiento guías de buenas prácticas (Yoshinaga-Itano, 2014).

Nombramos "Valoraciones de la tecnología de apoyo" al cuarto factor con tres ítems sobre la valoración de los recursos: apoyos visuales, pizarra digital y representaciones con imágenes obteniéndose una valoración media-baja. Aunque las tecnologías de apoyo generalmente se han aplicado a dispositivos electrónicos y hardware y software de los ordenadores, hay otros recursos disponibles, acogidos a la filosofía del Diseño Universal de Aprendizaje que aglutina modelos, métodos y productos que aumentan la experiencia educativa de estudiantes, entre otros, el sistema de audio FM personal que transmite la voz de un hablante directamente al oído de un usuario, o programas de reconocimiento de voz, audiolibros, etc. Así, Maidment, Kang, Stewart, y Amitay (2015, p. 61) exploraron si la información visual mejoraba la identificación del habla indicando que "los niños menores de 6 años de edad no utilizaban completamente las señales visuales del habla para mejorar la percepción del habla cuando la señal auditiva se degradaba" (por ejemplo, con un sintetizador de voz).

Las tecnologías anteriormente mencionadas constituyen la base de un sistema inteligente de eSalud y eCuidado, con las que se podrían resolver problemas en niños y adolescentes con dificultades auditivas derivados de la falta de atención en ambientes ruidosos (King, Boutilier, y Chorne, 2018).

\section{Limitaciones}

Este estudio ha soslayado observaciones sistemáticas y entrevistas a los niños y adolescentes de la muestra y ha privilegiado la cohesión cultural de las escuelas públicas que soportan la inclusión. Tampoco, ha analizado los lazos entre tipos de necesidades especiales y prácticas de clase o los resultados académicos de los estudiantes.

\section{Recomendaciones}

Los hallazgos de este estudio tienen implicaciones teóricas para comprender las actitudes que tienen los estudiantes con IC o AU de escuelas de educación inclusiva. Reflexionando sobre los factores hallados, se pueden planificar y desarrollar estrategias para la individualización de la instrucción y adaptar estándares de aprendizaje para estudiantes con dificultades auditivas, y paralelamente sugerir acciones formativas para el Prácticum de la titulación de Maestro. Se debe prestar una atención más estrecha a la esencia de la inclusión disminuyendo los muros de estándares y aumentando la participación en condiciones organizativas apropiadas bajo un enfoque explícito de igualdad.

\section{Conclusiones}

Los factores "Implicación familiar y opinión del uso de la tecnología”, "Inclusión en el Centro", "Comunicación con especialistas médicos" y "Valoraciones de la tecnología de apoyo" procedentes del CINA han sido desarrollados y validados con fiabilidad y validez satisfactorias. Este estudio tiene las características de un conocimiento base "emergente" de las actitudes de niños y adolescentes con dificultades auditivas medidas directamente por los mismos estudiantes. La media final de la actitud de los escolares hacia la inclusión ha sido alta. El factor "Inclusión en el Centro" ha tenido la media más elevada. La media de la actitud de los estudiantes con IC en el factor "Comunicación con especialistas médicos" ha sido la más baja. Existieron diferencias significativas entre estudiantes con IC y AU en los factores "Inclusión en el Centro" y "Valoraciones de la tecnología de apoyo".

\section{Referencias bibliográficas}

Alegre, O.M. y Villar, L.M. (2015). Inclusión e Interculturalidad. Un estudio en el marco de la enseñanza universitaria. Revista nacional e internacional de educación inclusiva, 8(3), 12-29.

Alegre, O.M, Rodríguez, M.C., Villar, L.M., y Pérez, D. (2016). Evaluación de la eficacia del Implante Coclear en función de la edad de implantación. European Scientific Journal,12(26), 4251. https://doi.org/:10.19044/esj.2016.v12n26p42.

Amiama, J.F., Ledesma, N., y Monzón, J. (2017). La participación del alumnado en proyectos educativos vinculados al territorio: propuestas inclusivas en un centro escolar de secundaria. Aula Abierta, 46, 91-96. https://doi.org/10.17811/rifie.46.2017.91-96

Antia, S.D., Sabers, D.L., y Stinson, M.S. (2007). Validity and Reliability of the Classroom Participation Questionnaire With Deaf and Hard of Hearing Students in Public Schools. Journal of Deaf Studies and Deaf Education, 12(2), 158-171. https://doi. org/10.1093/deafed/enl028.

Archbold, S., y Mayer, C. (2012). Deaf Education: The Impact of Cochlear Implantation? Deafness and Education International, 14, 2-15. https://doi.org/10.1179/1557069X12Y.0000000003.

Bakar, Z.A, Brown, P.M, y Remine, M.D. (2010). Sensitivity in Interactions between Hearing Mothers and their Toddlers with Hearing Loss: The Effect of Cochlear Implantation. Deafness and Education International, 12, 2-15. https://doi.org/10.1179/1 $46431510 \times 12626982043525$

Beaulieu-Bergeron, R., y Morin, D. (2016). A Qualitative Investigation of Fifth- and Sixth-grade Students' Attitudes towards Intellectual Disability. International Journal of Disability, Development and Education, 63(5), 514-528. https://doi.org/10.1080/ $1034912 X .2016 .1144874$.

Bellis, T.J. (2011). Assessment and Management of Central Auditory Processing Disorders in the Educational Setting: From Science to Practice. Second Edition. San Diego: Plural Publishing, USA.

Booth, T., y Ainscow, M. (2002). Index for Inclusion: Developing learning and Participation in Schools. Centre for Studies on Inclusive Education (CSIE). Bristol: England.

Bradham, T.S., y Houston, K.T. (2014). Assessing Listening and Spoken Language in Children with Hearing Loss. San Diego: Plural Publishing, USA.

Caldwell, A., y Nittrouer, S. (2013). Speech perception in noise by children with cochlear implants. Journal of Speech, Language, and Hearing Research, 56(1), 13-30. https://doi:10.1044/10924388/2012/11-0338.

Cepa, A., Heras, D., y Fernández-Hawryla, M. (2017). La educación emocional en la infancia: una estrategia inclusiva. Aula Abierta, 46, 73-82. https://doi.org/10.17811/rifie.46.2017.73-82.

Ching, T.Y.C., Scarinci, N., Marnane, V., Sjahalam-King, J., Button, L., y Whitfield, J. (2018). Factors influencing parents' decisions about communication choices during early education of their child with hearing loss: a qualitative study. Deafness $\mathcal{E}$ Education International, 20(3-4), 154-181. https://doi.org/ 10.1080/14643154.2018.1512393.

Chute, P.M., y Nevins, M.E. (2006). School professionals working with children with cochlear implants. San Diego, Plural Publishing, USA.

Davenport, C.A., y Alber-Morgan, S.R. (2016). I Have a Child With a Cochlear Implant in My Preschool Classroom. Now What? Teaching Exceptional Children, TEACHING Exceptional Children, 49(1), 41-48. https://doi.org/ 10.1177/0040059916651430.

Deng, M., Wang, S., Guan, W., y Wang, Y. (2017). The development and initial validation of a questionnaire of inclusive teachers' competency for meeting special educational 
needs in regular classrooms in China. International Journal of Inclusive Education, 21(4), 416-427. https://doi.org/ 10.1080/13603116.2016.1197326.

Fernández-López, J.A.; Fernández Fidalgo, M.; Cieza, A. Y Ravens-Sieberer, U. (2004). Medición de la calidad de vida en niños y adolescentes: comprobación preliminar de la validez y fiabilidad de la versión española del cuestionario KINDL. Atención Primaria, 33(8), 432-442.

Gale, E. (2011). Exploring Perspectives on Cochlear Implants and Language Acquisition Within the Deaf Community. Journal of Deaf Studies and Deaf Education, 16(1), 121-139. https://doi. org/10.1093/deafed/enq044.

Garberoglio, C.L., Gobble, M.E., y Cawthon, S.W. (2012). A National Perspective on Teachers' Efficacy Beliefs in Deaf Education. The Journal of Deaf Studies and Deaf Education, 17(3), 367-383. https://doi.org/10.1093/deafed/ens014.

Geers, A.E., Nicholas, J., Tobey, E., y Davidson, L. (2016). Persistent language delay versus late language emergence in children with early cochlear implantation. Journal of Speech, Language, and Hearing Research, 59, 155-170. https://doi.org/ 10.1044/2015_JSLHR-H-14-0173.

Grobler, H.B., y Wessels, S. (2018). Hear Their Voices: Self-configuration Experiences of Learners with Mild Learning Difficulties within the Learner-Teacher Relationship. International Journal of Disability, Development and Education. https://doi. org/10.1080/1034912X.2018.1499878.

He, J.P., Burstein, M., Schmitz, A., y Merikangas, K.R. (2013). The Strengths and Difficulties Questionnaire (SDQ): The Factor Structure and Scale Validation in U.S. Adolescents. Journal of Abnormal Child Psychology, 41(4), 583-595. https://doi. org/10.1997/s10802-012-9696-6.

Hintermair, M., y Albertini, J.A. (2005). Ethics, Deafness, and New Medical Technologies. The Journal of Deaf Studies and Deaf Education, 10(2), 184-192. https://doi.org/10.1093/deafed/eni018.

Holt, R.F., Beer, J., Kronenberger, W.G., Pisoni, D.B., y Lalonde, K. (2012). Contribution of Family Environment to Pediatric Cochlear Implant Users' Speech and Language Outcomes: Some Preliminary Findings. Journal of Speech, Language, and Hearing Research, 55(3), 848-864. https://doi.org/ 10.1044/10924388/2011/11-0143/.

Hung, H.-L., y Paul, P.V. (2006). Inclusion of Students who are Deaf or Hard of Hearing: Secondary School Hearing Students' Perspectives. Deafness $\mathcal{E}$ Education International, 8(2), 62-74. https://doi.org/10.1179/146431506790560229.

Hyde, M., y Punch, R. (2011). The modes of communication used by children with cochlear implants and the role of sign in their lives. American Annals of the Deaf, 155(5), 535-49. https:// doi.org/10.1353/aad.2011.006.

Kecman, E. (2018). Old challenges, changing contexts: reviewing and reflecting on information provision for parents of children who are deaf or hard-of-hearing. Deafness $\mathcal{E}$ Education International. https://doi.org/ 10.1080/14643154.2018.1506072.

King, S., Boutilier, J.A., y Chorne, J.M. (2018). Managing Chronic Pain in the Classroom: Development and Usability Testing of an eHealth Educational Intervention for Educators. $\mathrm{Ca}$ nadian Journal of School Psychology, 33(2), 95-109. https://doi. org/10.1177/0829573516674308.

McAloney-Kocaman, K., y McPherson, K. (2017). Factor Structure and Reliability of the Parent-Informant Strengths and Difficulties Questionnaire in a Scottish Preschool Sample. Early Education and Development, 28(3), 368-376. https://doi.org/10.1 080/10409289.2017.1228367.

Maidment, D.W., Kang, H.J., Stewart, H.J., y Amitay, S. (2015). Audiovisual integration in children listening to spectra- lly degraded speech. Journal of Speech, Language, and Hearing Research, 58(1), 61-8. https://doi.org/ 10.1044/2014_JSLHR-S-14-0044.

Mayer, C., y Trezek, B.J. (2018). Literacy Outcomes in Deaf Students with Cochlear Implants: Current State of the Knowledge. Journal of Deaf Studies and Deaf Education, 23(1), 1-16. https://doi.org/10.1093/deafed/enx043.

Meinzen-Derr, J., Wiley, S., Phillips, J., Altaye, M., y Choo, D.I. (2017). The utility of early developmental assessments on understanding later nonverbal IQ in children who are deaf or hard of hearing. International Journal of Pediatric Otorhinolaryngology, 92, 136-142. https://doi.org/10.1016/j. ijporl.2016.11.024.

Michael, R., Cinamon, R.G., y Most T. (2015). What Shapes Adolescents' Future Perceptions? The Effects of Hearing Loss, Social Affiliation, and Career Self-Efficacy. The Journal of Deaf Studies and Deaf Education, 20(4), 399-407. https://doi. org/10.1093/deafed/env023.

Moeller, M.P., Carr, G., Seaver, L., Stredler-Brown, A., y Holzinger, D. (2013). Best Practices in Family-Centered Early Intervention for Children Who Are Deaf or Hard of Hearing: An International Consensus Statement. Journal of Deaf Studies and Deaf Education, 18(4), 429-445. https://doi.org/10.1093/deafed/ ent034.

Moore, B.C.J. (2007). Cochlear Hearing Loss: Physiological, Psychological and Technical Issues, Second Edition. Online. John Wiley \& Sons Ltd. https://doi.org/10.1002/9780470987889.

Most, T., Wiesel, A., y Blitzer, T. (2007). Identity and Attitudes towards Cochlear Implant Among Deaf and Hard of Hearing Adolescents. Deafness \& Education International, 9(2), 68-82. https://doi.org/10.1179/146431507790560002.

Mouvet, K., Matthijs, L., Loots, G., Taverniers, M., y Van Herreweghe, M. (2013). The Language Development of a Deaf Child with a Cochlear Implant. Language Sciences, 35, 59-79. https://doi.org/10.1016/j.langsci.2012.05.002.

Navarro, J. S., \& Merino, C. (2016). Estructura Latente del Kiddo-Kindl en una muestra Peruana. Liberabit,22(1), 77-89.

Noble, W. (2013). Self-assessment of Hearing. Second edition. San Diego: Plural Publishing, USA.

Nowicki, E.A., y Sandieson, R. (2002). A Meta-Analysis of SchoolAge Children's Attitudes Towards Persons with Physical or Intellectual Disabilities, International Journal of Disability, Development and Education, 49(3), 243-265. https://doi.org/10.108 0/1034912022000007270.

Radici, E., Heboyan, V., Mantovani, F., y De Leo, G. (2018). Teachers' Attitudes Towards Children Who Use AAC in Italian Primary Schools. International Journal of Disability Development and Education. https://doi.org/10.1080/103491 2X.2018.1495321.

Rekkedal, A.M. (2012). Assistive Hearing Technologies Among Students With Hearing Impairment: Factors That Promote Satisfaction. Journal of Deaf Studies and Deaf Education, 17(4), 499-517. https://doi.org/10.1093/deafed/ens023.

Roberts, C.M., y Lindsell, J.S. (1997). Children's Attitudes and Behavioural Intentions Towards Peers with Disabilities. International Journal of Disability, Development and Education, 44(2), 133-145. https://doi.org/10.1080/0156655970440205.

Scott, J.A., y Kasun, G.S. (2018). It's not enough to move your hands beautifully': teaching and learning at a school for deaf students in Mexico. International Journal of Bilingual Education and Bilingualism. https://doi.org/10.1080/13670050.2018.15457 44.

Schick, B., Skalicky, A., Edwards, T., Kushalnagar, P., Topolski, T., y Patrick, D. (2013). School placement and perceived qua- 
lity of life in youth who are deaf or hard of hearing. The Journal of Deaf Studies and Deaf Education, 18(1), 47-61. https:// doi.org/10.1093/deafed/ens039.

Sharma, U., Jitoko, F., Macanawai, S.S., y Forlin, Ch. (2018). How Do we Measure Implementation of Inclusive Education in the Pacific Islands? A Process for Developing and Validating Disability-Inclusive Indicators. International Journal of Disability, Development and Education, 65(6), 614-630. https://doi.org/ 10.1080/1034912X.2018.1430751.

Thurstone, L.L. (1928). Attitudes can be measured. American Journal of Sociology, 33, 529-554. http://dx.doi.org/10.1086/214483.

Tomblin, J.B., Barker, B.A., Spencer, L.J., Zhang, X., y Gantz, B.J. (2005). The effect of age at cochlear implant initial stimulation on expressive language growth in infants and toddlers. Journal of Speech, Language, and Hearing Research, 48(4), 853-67.

Van Gent, T., Goedhart, A.W., Knoors, H.E., Westenberg, P.M., y Treffers, P.D. (2012). Self-concept and ego development in deaf adolescents: a comparative study. The Journal of Deaf Studies and Deaf Education, 17(3), 333-51. https://doi. org/10.1093/deafed/ens002.
Wiley, S., Arjmand, E., Meinzen-Derr, J., y Dixon, M. (2011). Findings from multidisciplinary evaluation of children with permanent hearing loss. International Journal of Pediatric Otorhinolaryngology, 75(8), 1040-1044. https://doi.org/10.1016/j. ijporl.2011.05.019.

Woolf, S.B. (2018). Critical Skills for Special Educator Effectiveness: Which Ones Matter Most, and to Whom? Teacher Education and Special Education, 1-15. https://doi. org/10.1177/0888406418776714.

Yong, A.G., y Pearce, S. (2013). A Beginner's Guide to Factor Analysis: Focusing on Exploratory Factor Analysis. Tutorials in Quantitative Methods for Psychology, 9(2), 79-94. https://doi. org/10.20982/tqmp.09.2.p079.

Yoshinaga-Itano, Ch. (2014). Principles and Guidelines for Early Intervention After Confirmation That a Child Is Deaf or Hard of Hearing. The Journal of Deaf Studies and Deaf Education, 19(2), 143-175. https://doi.org/10.1093/deafed/ent043. 
